Sireeratawong et al., Afr J Tradit Complement Altern Med. (2013) 10(2):246-250

http://dx.doi.org/10.4314/ajtcam.v10i2.8

\title{
EVALUATION OF ANTI-INFLAMMATORY AND ANTINOCICEPTIVE ACTIVITY OF TRIPHALA RECIPE
}

\section{Seewaboon Sireeratawong ${ }^{1 *}$, Kanjana Jaijoy ${ }^{2}$, Noppamas Soonthornchareonnon ${ }^{3}$}

${ }^{1}$ Division of Pharmacology, Department of Preclinical Science, Faculty of Medicine, Thammasat University, Rungsit Campus, Klong Loung, Pathum Thani 12120, Thailand. ${ }^{2}$ Department of Pharmacology, Faculty of Medicine, Chiang Mai University, Chiang Mai 50200, Thailand. ${ }^{3}$ Department of Pharmacognosy, Faculty of Pharmacy, Mahidol University, Bangkok 10400, Thailand. Seewaboon Sireeratawong

*E-mail: seewaboon@gmail.com

\begin{abstract}
The anti-inflammatory and antinociceptive activities of Triphala recipe were studied in animal models. Triphala recipe (4 mg/ear) significantly exhibited an inhibitory effect on the ear edema formation induced by ethyl phenylpropiolateinduced, but not on the arachidonic acid -induced ear edema in rats. Furthermore, Triphala recipe at the doses of 300, 600 and $1,200 \mathrm{mg} / \mathrm{kg}$ significantly reduced carrageenan-induced hind paw edema. Next, the anti-inflammatory action in chronic inflammation was measured using the cotton pellet-induced granuloma formation assay in rats. Triphala recipe $(1,200 \mathrm{mg} / \mathrm{kg})$ reduced neither transudative weight nor granuloma formation. It also did not affect on body weight gain and thymus weight indicating that Triphala recipe does not have a steroid-like effect. In antinociceptive study, Triphala recipe (300, 600, 1,200 $\mathrm{mg} / \mathrm{kg}$ ), elicited significant inhibitory effect on both phases, especially in late phase, of the formalin test in mice suggesting that the antinociceptive action of Triphala recipe may be via both peripheral and at least partly centrally acting.
\end{abstract}

Keywords: anti-inflammatory, antinociceptive, Triphala recipe

\section{Introduction}

Triphala has commonly been used in an Ayurvedic and Thai traditional medicine. It consists of the dried fruits of three plants, Phyllanthus emblica Linn., Terminalia chebula Retz., and Terminalia bellerica (Gaertn.) Roxb. Triphala recipe is a botanical preparation comprised of equal proportions of the three herbal fruits. It is used for adjusting the balance of four elements of the body and detoxifying the body systems, especially the gastrointestinal system, blood and lymph systems. Thai traditional medicine doctors use this recipe to removing waste products (i.e. fecal matter, urine and sweat).

Triphala recipe possesses many biological activities including antimutagenic (Kaur et al., 2002), radioprotective (Sandhya et al., 2006a), hypocholesterolaemic (Saravanan et al., 2007), immunomodulatory (Srikumar et al., 2005), anticancer (Deep et al., 2005; Sancheti et al., 2005; Sandhya and Mishra, 2006; Sandhya et al., 2006b), and anti-oxidant activities (Naik et al., 2005). Moreover, this recipe has an anti-inflammatory activity on adjuvant-induced arthritis in mice (Rasool and Sabina, 2007).

However, there are a few reports regarding the anti-inflammatory and antinociceptive activities of this recipe. The objective of this study was thus to evaluate the anti-inflammatory and antinociceptive activities of Triphala recipe using animal models.

\section{Materials and Methods \\ Preparation of Triphala recipe}

In our laboratory, a decoction of each plant raw materials (P. emblica, T. chebula and T. bellerica) was developed as follows: the raw material was immersed in 100 liters of water for $30 \mathrm{~min}$, then boiled for 1 hour and filtered to remove the residue. Next, the aqueous extract was repeatedly boiled and filtered twice. Next, spray drying process was carried out to remove any residual trace of the extraction solvent in the extract. The extract was standardized by thin layer chromatography (TLC) and high performance liquid chromatography (HPLC). Each aqueous extract was stored at $-20^{\circ} \mathrm{C}$ until the preparation of Triphala recipe. Triphala recipe was further prepared from a mixture containing one kilogram of each extract.

\section{Experimental animals}

Male ICR albino mice (30-40 g) and male Sprague Dawley rats (40-60 g, 100-120 g, 200-220 g) were procured from the National Laboratory Animal Center, Nakorn Pathom, Thailand. The animals were housed a temperature-controlled room $\left(25 \pm 1{ }^{\circ} \mathrm{C}\right)$ and provided with standardized pelleted feed and clean drinking water ad libitum. The study has got the clearance from the Animal Ethics Committee of Faculty of Medicine, Thammasat University, Pathum Thani, Thailand (No. 0001/2008). 


\section{Ethyl phenylpropiolate (EPP) or arachidonic acid (AA)-induced ear edema in rats}

Male rats (40-60 g) were used and the ear edema was produced by topical application of EPP ( $1 \mathrm{mg} / 20 \mu \mathrm{l} / \mathrm{ear})$ or AA $(2 \mathrm{mg} / 20 \mu \mathrm{l} / \mathrm{ear})$ to the inner and outer surface of both ears. Triphala recipe (4 mg/ear), phenylbutazone ( $1 \mathrm{mg} / \mathrm{ear})$, phenidone (2 mg/ear) and vehicle (the mixture of dimethysulfoxide and acetone, 1:1) were applied in the same manner in a volume of $20 \mu \mathrm{l}$ just before the irritants. The edema thickness was measured with digital vernier calipers at $0,15,30,60$, and 120 min after EPP induction and at 60 min after AA induction.

\section{Carrageenan-induced paw edema in rats}

Five groups of male rats (100-120 g) containing six animals per group orally received distilled water (control, 2 $\mathrm{ml} / \mathrm{kg})$, Triphala recipe $(300,600$ and $1,200 \mathrm{mg} / \mathrm{kg}$ ) and aspirin $(300 \mathrm{mg} / \mathrm{kg})$. One hour after orally administration of test substance, acute inflammation was produced by an intradermal injection of carrageenan (1\% in normal saline solution, NSS) into the plantar surface of the right hind paw of the rat at a volume of $0.05 \mathrm{ml}$. The paw edema volume was measured using a plethysmometer (model 7140, Ugo Basile, Italy) at 0, 1, 3 and 5 hours after carrageenan injection.

\section{Cotton pellet-induced granuloma formation in rats}

Four groups of male rats (200-220 g, $\mathrm{n}=6$ ) were used. The distilled water (control, $2 \mathrm{ml} / \mathrm{kg}$ ), aspirin (300 mg/kg), prednisolone $(5 \mathrm{mg} / \mathrm{kg})$ and Triphala recipe $(1,200 \mathrm{mg} / \mathrm{kg})$ were orally given to the rats. One hour after, the animals under ether anesthesia were implanted subcutaneously with two sterilized cotton pellets (20 $\pm 1 \mathrm{mg})$, one on each side of the abdomen. The test substances were administered orally in a once daily dosage regimen throughout the experimental period of 7 days. On the eighth day, each rat was anaesthetized with thiopental sodium (40 mg/kg). The cotton pellets and thymus were removed, dried at $60{ }^{\circ} \mathrm{C}$ for $18 \mathrm{~h}$ and their dry weight determined. The change in body weight from the first and the last day of experiment was also recorded. The transudative and granuloma weight, as well as the percent granuloma inhibition of the test drugs were calculated.

\section{Formalin test}

Male ICR mice were used and divided into 6 groups and treated respectively with Triphala (300, 600, 1,200 $\mathrm{mg} / \mathrm{kg}$ ), aspirin (300 mg/kg), and distilled water (control, $1 \mathrm{ml} / \mathrm{kg}$ ). One hour later, $1 \%$ formalin in NSS (20 $\mu \mathrm{l}$ ) was injected subcutaneously into the left hand foot pad, immediately placed in a transparent plastic cage separately; the licking time were recorded for $5 \mathrm{~min}$ after formalin injection for early phase. In the late phase assessment, another set of mice were used. The formalin was injected $40 \mathrm{~min}$ after oral administration of test substance and the licking time was determined between 20 and 30 min after the injection of formalin

\section{Statistical analysis}

Results were expressed as mean \pm standard error of mean (S.E.M.). Statistical significance was determined by oneway analysis of variance (ANOVA) and post hoc least-significant difference (LSD) test. $P$ values less than 0.05 were considered significant.

\section{Results and discussion}

The inhibitory effect of Triphala recipe on EPP-induced ear edema is shown in Figure 1A. Inflammation in EPP model involves instant irritation which leads to fluid accumulation and edema characteristic. The inflammatory mediators released in this model are histamine, serotonin, bradykinin and prostaglandins (PGs), and these mediators are capable of promoting vasodilatation and increasing vascular permeability as well as producing edema (Brattsand et al., 1982; Carlson et al., 1985). Triphala recipe (4 mg/ear) and phenylbutazone $(1 \mathrm{mg} / \mathrm{ear})$ significantly reduced the edema thickness at all assessment times.

AA-induced ear edema is an inflammation model which is used to evaluate the anti-inflammatory activity of lypoxygenase inhibitors (Young et al., 1984; Opas et al., 1985). AA causes an increase in $\mathrm{LTB}_{4}, \mathrm{LTC}_{4}$, and PGE $\mathrm{P}_{2}$ contents in ear tissue (Horizoe et al., 1998) and especially LOX metabolites which are major mediators of the inflammatory process (Chang et al., 1986; Di Martino et al., 1987). Triphala recipe (4 mg/ear) showed no inhibitory effect on AA-induced ear edema (Figure 1B). Since Triphala recipe exhibited an inhibitory effect on the ear edema formation induced by EPP, but not on the AA-induced ear edema, these indicate the anti-inflammatory activity of Triphala recipe does not involve the lipoxygenase pathway. 

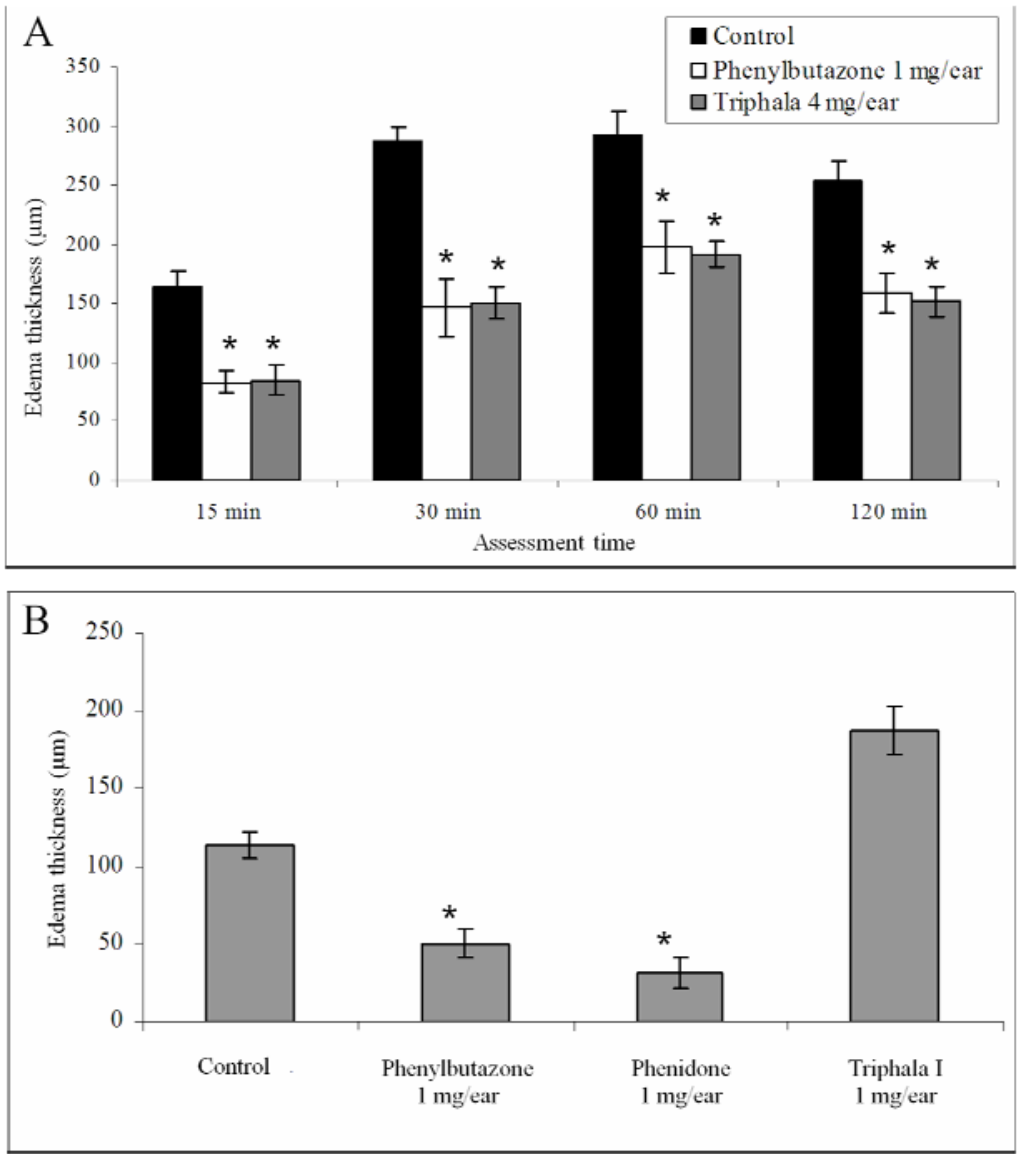

Figure 1 Inhibitory effect of Triphala recipe on edema thickness of EPP (A) or AA (B) induction in rats. *Significantly different from the control group, $p<0.05$.

Carrageenan-induced hind paw edema is successfully used for identifying inhibitors of cyclooxygenase (Winter et al., 1962). Inflammatory formation of carrageenan involves two distinct phases of mediator release. Histamine, serotonin and bradykinin are released in first phase which occurs between 0 and $2 \mathrm{~h}$ after carrageenan injection. In late phase, the inflammation reaches it maximum approximately $3 \mathrm{~h}$ after carrageenan injection and then it begins to decline. The releasing of prostaglandin in late phase is the cause of edema which depends on the mobilization of neutrophils (Di Rosa et al., 1971). In our study, Triphala recipe at the doses of 300,600 and $1,200 \mathrm{mg} / \mathrm{kg}$ significantly reduced the paw edema volume at all assessment times when compared with the control group as shown in Figure 2. The inhibitory effect of Triphala recipe on carrageenan-induced paw edema at the $3^{\text {rd }}$ hour suggests that the action of Triphala recipe may involve the inhibition of the synthesis or the release of prostaglandins.

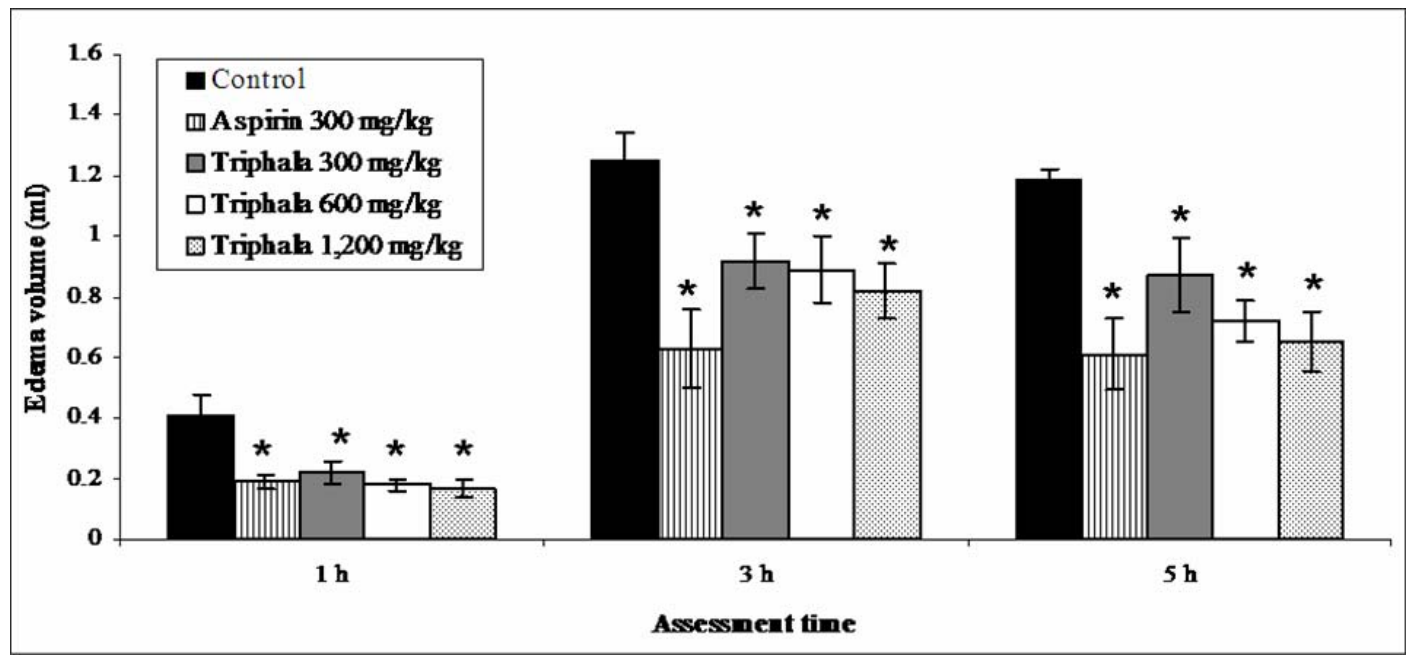

Figure 2: Inhibitory effect of Triphala recipe on paw edema volume of carrageenan induction in rats 
Anti-inflammatory action in chronic inflammation was demonstrated by the ability to inhibit the increase of number of fibroblasts during granuloma tissue formation (Gupta et al., 2003). In this experiment, Triphala recipe did not reduce transudative weight and granuloma formation (Table 2). The obtained result suggests that Triphala recipe does not possess the inhibitory effect on granuloma formation. In addition, Triphala recipe did not affect on body weight gain and thymus weight indicating that Triphala recipe does not have a steroid-like effect (Table 3).

Table 2: Effect of Triphala recipe on granuloma formation and transudation in cotton pellet-induced granuloma formation in

\begin{tabular}{lccc}
\multicolumn{1}{c}{ Group } & Transudative weight (mg) & $\begin{array}{c}\text { Granuloma weight } \\
\text { (mg/mg cotton) }\end{array}$ & GI (\%) \\
\hline 5\% Tween80 & $427.39 \pm 50.21$ & $3.55 \pm 0.41$ & - \\
Prednisolone $5 \mathrm{mg} / \mathrm{kg}$ & $263.40 \pm 17.10^{*}$ & $2.59 \pm 0.25^{*}$ & 27 \\
Aspirin $300 \mathrm{mg} / \mathrm{kg}$ & $339.52 \pm 17.82$ & $2.97 \pm 0.24$ & 16 \\
Triphala $1,200 \mathrm{mg} / \mathrm{kg}$ & $340.92 \pm 24.37$ & $2.87 \pm 0.19$ & 19 \\
\hline
\end{tabular}

Data represent mean \pm S.E.M. $(n=6)$.

*Significantly different from the control group, $p<0.05$

$\mathrm{GI}$ = granuloma inhibition

Table 3: Effect of Triphala recipe on body weight and thymus weight in cotton pellet-induced granuloma formation in rats

\begin{tabular}{lcccc}
\hline \multicolumn{1}{c}{ Group } & \multicolumn{3}{c}{ Body weight (gram) } & \multirow{2}{*}{$\begin{array}{c}\text { Dry thymus weight } \\
\text { (mg/100g) }\end{array}$} \\
\cline { 2 - 4 } & Initial & Final & Gain & $2.2 \pm 1.4$ \\
5\% Tween80 & $384.2 \pm 20.5$ & $392.5 \pm 20.1$ & $8.33 \pm 2.47$ & $2 . .^{*}$ \\
Prednisolone $5 \mathrm{mg} / \mathrm{kg}$ & $357.5 \pm 115$ & $349.2 \pm 11.8^{*}$ & $-8.33 \pm 4.94^{*}$ & $17.1 \pm 0.8^{*}$ \\
Aspirin $300 \mathrm{mg} / \mathrm{kg}$ & $368.3 \pm 13.8$ & $365.0 \pm 12.2$ & $-3.33 \pm 2.79$ & $19.2 \pm 1.3$ \\
Triphala $1,200 \mathrm{mg} / \mathrm{kg}$ & $361.7 \pm 12.4$ & $358.3 \pm 15.4$ & $-3.33 \pm 7.49$ & $23.5 \pm 1.1$ \\
\hline
\end{tabular}

Data represent mean \pm S.E.M. $(n=6)$.

*Significantly different from the control group, $p<0.05$

The formalin test is a well described model of nociception which consisted of two distinct phases. The nociception in early phase is due to the direct stimulation of the sensory nerve fibers by formalin which can be inhibited by centrally acting antinociceptives (Hunskaar et al., 1985; Hunskaar and Hole, 1987). The late phase response is due to inflammatory mediators such as histamine, prostaglandins, serotonin and bradykinin (Murray et al. 1988, Tjolsen et al. 1992, Dharmasiri et al. 2003). This phase can be inhibited by NSAIDs (aspirin, indomethacin and naproxen) and steroids (dexamethasone and hydrocortisone) as well as the centrally acting drugs (Hunskaar and Hole, 1987).

In the present study, the licking of foot was triggered by subcutaneous injection of formalin into the hand foot pad. Triphala recipe (300, 600, 1,200 mg/kg), aspirin (300 mg/kg) and morphine (10 mg/kg) elicited significant inhibitory effect on both phases of the formalin test in mice, especially in late phase as shown in Figure 3. The antinociceptive activity of Triphala recipe in the early phase may be the centrally acting mechanism. The activity of Triphala recipe found in late phase rather indicates the inhibitory effect on the synthesis and/or release of inflammatory pain mediators especially prostaglandins.

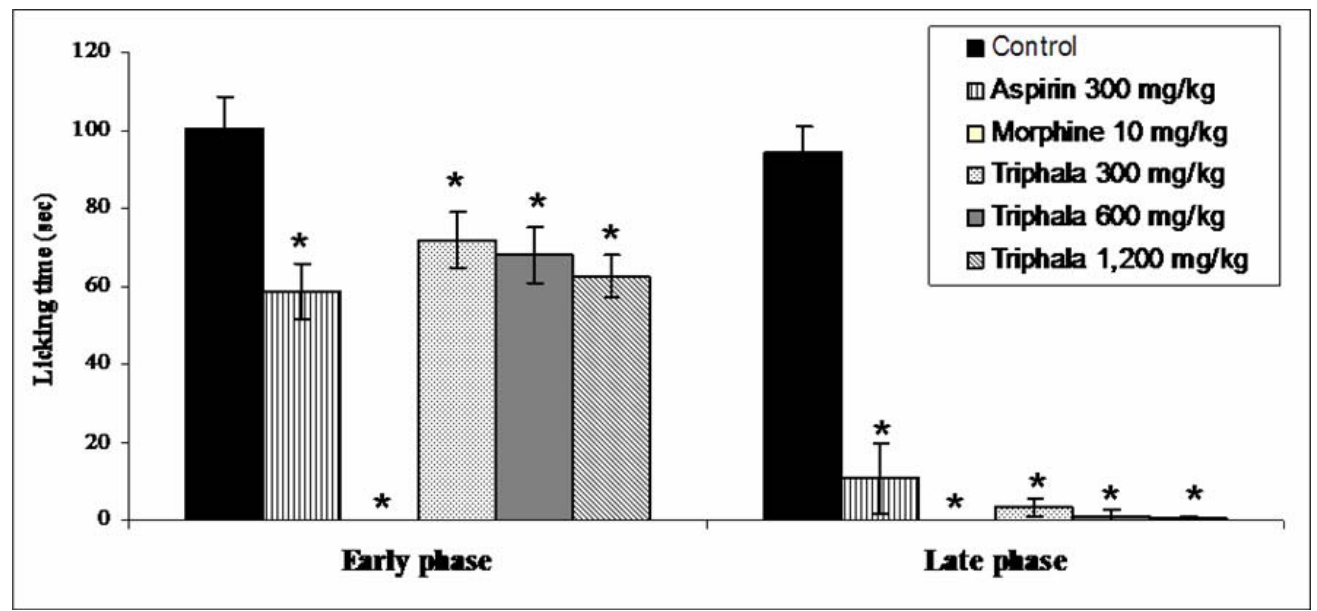

Figure 3: Inhibitory effect of Triphala recipe on licking response of formalin test in mice

In conclusion, Triphala recipe has both the anti-inflammatory and antinociceptive activities. The former effect is perhaps mediated via inhibition of cyclooxygenase pathway, and free of steroidal-like action. The antinociceptive activity of Triphala recipe may be via both peripheral acting and partly central acting. 


\section{Acknowledgements}

The authors would like to thank the National Research Council of Thailand for financial support.

\section{References}

1. Brattsand, R., Thalen, A., Roempke, K., Kallstrom, L. and Gruvstad, E. (1982). Influence of 16 alpha, 17 alphaacetal substitution and steriod nucleus fluorination on the topical to systemic activity ratio of glucocorticoids. J. Steroid. Biochem. 16(6): 779 - 786.

2. Carlson, R.P., O’Neill-Davis, L., Chang, J. and Lewis, A.J. (1985). Modulation of mouse ear edema by cyclooxygenase and lipoxygenase inhibitors and other pharmacological agents. Agents Actions. 17: 197 - 204.

3. Chang, J., Carlson, R.P., O’Neill-Davis, L., Lamb, B., Sharma, R.N. and Lewis, A.J. (1986). Correlation between mouse skin inflammation induced by arachidonic acid and eicosanoid synthesis. Inflammation. 10(3): 205 - 214.

4. Dharmasiri, J.R., Jayakody, A.C., Galhena, G., Liyanage S.S.P. and Ratnasooriya, W.D. (2003). Anti-inflammatory and analgesic activities of mature fresh leaves of Vitex negundo. J. Ethnopharmacol. 87: 199 - 206.

5. Deep, G., Dhiman, M., Rao, A.R. and Kale, R.K. (2005). Chemopreventive potential of Triphala (a composite Indian drug) on benzo(a)pyrene induced forestomach tumorigenesis in murine tumor model system. J. Exp. Clin. Cancer Res. 24(4): 555 - 563.

6. Di Martino, M.J., Campbell, G.K., Wolff, C.E. and Hanna, N. (1987). The pharmacology of arachidonic acid induced rat paw edema. Agents Actions. 21: 303 - 305.

7. Di Rosa, M., Giroud, J.P. and Willoughby, D.A. (1971). Studies on the mediators of the acute inflammatory response induced in rats in different sites by carrageenan and turpentine. J. Pathol. 104(1): 15 - 29.

8. Gupta, M., Mazumder, U., Ramanathan, S.K. and Thangavel, S.K. (2003). Studies on anti-inflammatory, analgesic and anti-pyretic properties of methanol extract of Caesalpinia bonducella leaves in experimental animal models. Iran J. Pharmacol. Therapeut. 2: 30 - 34.

9. Horizoe, T., Nagakura, N., Chiba, K., Shirota, H., Shinoda, M., Kobayashi, N., Numata, H., Okamoto, Y. and Kobayashi, S. (1998). ER-34122, a novel dual 5-lipoxygenase/cyclooxygenase inhibitor with potent antiinflammatory activity in an arachidonic acid-induced ear inflammation model. Inflamm. Res. 47(10): 375 - 383.

10. Hunskaar, S., Fasmer, O.B. and Hole, K. (1985). Formalin test in mice, a useful technique for evaluating mild analgesic. J. Neurosci. Methods. 14(1): 69 - 76.

11. Hunskaar, S. and Hole, K. (1987). The formalin test in mice: dissociation between inflammatory and noninflammatory pain. Pain. 30(1): 103 - 114.

12. Kaur, S., Arora, S., Kaur, K. and Kumar, S. (2002). The in vitro antimutagenic activity of Triphala-an Indian herbal drug. Food Chem. Toxicol. 40(4): 527 - 534.

13. Murray, C.W., Porreca, F. and Cowan, A. (1988). Methodological refinements in the mouse paw formalin test an animal model of tonic pain. J. Pharmacolog. Methods. 20: 175 - 186.

14. Naik, G.H., Priyadarsini, K.I., Bhagirathi R.G., Mishra, B., Mishra, K.P., Banayalikar, M.M. and Mohan, H. (2005). In vitro antioxidant studies and free radical reactions of triphala, an ayurvedic formulation and its constituents. Phytother. Res. 19(7): 582 - 586.

15. Opas, E.E., Bonney, R.J. and Humes, J.L. (1985). Prostaglandin and leukotriene synthesis in mouse ears inflamed by arachidonic acid. J. Invest. Dermatol. 84(4): 253 - 256.

16. Rasool, M. and Sabina, E.P. (2007). Antiinflammatory effect of the Indian Ayurvedic herbal formulation Triphala on adjuvant-induced arthritis in mice. Phytother. Res. 21(9): 889 - 894.

17. Sancheti, G., Jindal, A., Kumari, R. and Goyal, P.K. (2005). Chemopreventive action of Emblica officinalis on skin carcinogenesis in mice. Asian Pac. J. Cancer Prev. 6(2): 197 - 201.

18. Sandhya, T., Lathika, K.M., Pandey, B.N., Bhilwade, H.N., Chaubey, R.C., Priyadarsini, K.I. and Mishra, K.P. (2006a). Protection against radiation oxidative damage in mice by Triphala. Mutat. Res. 609(1): 17 - 25.

19. Sandhya, T., Lathika, K.M., Pandey, B.N. and Mishra, K.P. (2006b). Potential of traditional ayurvedic formulation, Triphala, as a novel anticancer drug. Cancer Lett. 231(2): 206 - 214.

20. Sandhya, T. and Mishra, K.P. (2006). Cytotoxic response of breast cancer cell lines, MCF 7 and T 47 D to triphala and its modification by antioxidants. Cancer Lett. 238(2): 304 - 313.

21. Saravanan, S., Srikumar, R., Manikandan, S., Jeya Parthasarathy, N. and Sheela Devi, R. (2007). Hypolipidemic effect of triphala in experimentally induced hypercholesteremic rats. Yakugaku Zasshi. 127(2): 385 - 388.

22. Srikumar, R., Jeya Parthasarathy, N. and Sheela Devi, R. (2005). Immunomodulatory activity of triphala on neutrophil functions. Biol. Pharm. Bull. 28(8): 1398 - 1403.

23. Tjolsen, A., Berge, O.G., Hunskaar, S., Rosland, J.H. and Hole, K. (1992). The formalin test: an evaluation of the method. Pain. 51(1): 5 - 17.

24. Winter, C.A., Risley, E.A. and Nuss, G.W. (1962). Carrageenin-induced edema in hind paw of the rat as an assay for anti-inflammatory drug. Proc Soc Exp. Biol. Med. 111: 544 - 547.

25. Young, J.M., Spires, D.A., Bedord, C.J., Wagner, B., Ballaron, S.J. and De Young, L.M. (1984). The mouse ear inflammatory response to topical arachidonic acid. J. Invest. Dermatol. 82(4): 367 - 371. 